\title{
Handling Catastrophic Failures in Scalable Internet Applications
}

\author{
Michael Haungs
}

\author{
Raju Pandey, Earl Barr
}

\begin{abstract}
User perceived quality is the most important aspect of Internet applications. After a single negative experience, users tend to switch to one of the other myriad of alternatives available to them on the Internet. Two key components of Internet application quality are scalability and reliability. In this paper, we present the first general-purpose mechanism capable of maintaining reliability in the face of process, machine, and catastrophic failures. We define catastrophic failures as events that cause entire clusters of servers to become unavailable such as network partitioning, router failures, natural disasters, or even terrorist attacks. Our mechanism utilizes client-side tunneling, clientside redirection, and implicit redirection triggers to deliver reliable communication channels. We capitalize on previous work, Redirectable Sockets (RedSocks), that focuses on Internet application scalability. RedSocks are communication channels enhanced with a novel session layer aimed at modernizing network communication. We modify RedSocks to create the first fault tolerant socket solution that can handle all server-side failures. Our mechanism is compatible with NATs and Firewalls, scalable, application independent, and backwards compatible.
\end{abstract}

\section{Introduction}

It is crucial for Internet applications to be available 24-7. One study found that two-thirds of Internet users will rarely return to a site after a single bad experience [10]. Bhatti et al. [2] state, "Users have too many web sites that they can use as alternatives if they are either refused entry to one site or are given particulary slow service." For some popular web services, a single server failure can result in tens of thousands of lost customers.

To achieve 24/7 availability, Internet applications need to be scalable and reliable. The most popular solution to scalability is to construct multiple clusters of commodity servers and route incoming requests to them via a mechan- ism such as DNS Round Robin [3] or URL-rewriting [8]. L4 or L7 switches are then used for intracluster load balancing. No standard solution exists for making Internet applications reliable, but researchers have proposed a number of ideas $[1,12,13,9]$. One reason for the lack of a standard solution for reliability is the tension that exists between reliability and scalability. Scalable systems are more complex or introduce central failure points which increases the vulnerability of the server system. At the same time, overly cautious failover mechanisms directly affect system scalability by increasing server response latency.

Current approaches to Internet application reliability fall into two categories. The first category, connection redirection, insures new connections reach a healthy server. Both DNS and network switches, such as Cisco's LocalDirector [4], can be configured to incorporate server health information in their connection routing. Connection redirection complements the second category, communication fault tolerance. The goal here is to allow existing communication channels to persist in the face of machine failure. Most solutions use primary-backup or log-based recovery.

However, no solution addresses catastrophic failures. We define catastrophic failures as failures that completely block all communication from the current server cluster to the client. These failures can result from accidently cut transmission lines, router hardware failures, natural disasters, or even terrorist attacks. After a catastrophic failure, only the client and a route to an alternate server cluster exists. From this, the existing communication channel must be reconnected to an alternate cluster and all communication state and associated application state reconstructed.

We define a fault tolerant socket as a communication channel between two applications, typically a client and a server, that persists in the face of a process, machine, or catastrophic failure. In Figure 1, we illustrate where failures can occur along a communication channel and put them into two categories: server-side and client-side. Attempts in increasing server application reliability often target process (A) and machine (B) failures. Using a redundant router handles an error at the entry point (D) to the Internet ap- 
plication. Cut cables or earthquakes are examples of events that can cause the complete failure of server clusters (C) and associated entry points (D). There are two types of failures that occur in the Internet outside the server's administrative domain. The first are errors that can be circumvented via normal IP routing recovery $(\mathrm{E})$. The second $(\mathrm{F})$ occurs when there is no alternative path from the client to the origin server cluster. We categorize failures at points A through $\mathrm{F}$ as server-side errors. Fault tolerant sockets must handle all server-side failures. Client-side failures (H, I, and J in Figure 1) are beyond the scope of Internet server applications. Put another way, fault tolerant sockets persist as long as the client does.

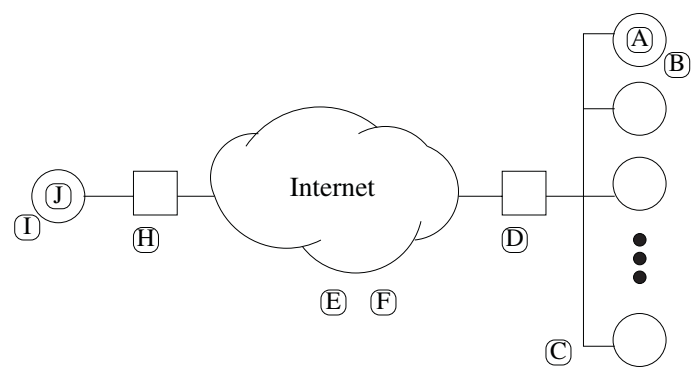

Figure 1. Various failure points along a client/server communication channel.

To handle server-side errors, a failover mechanism must be able to detect that a failure occured, recover in-flight data, construct a new communication channel to an operational server, and provide a method to synchronize communication and application state. It must do this in a scalable way to be practical. Last, it must be compatible with methods for handling new connection redirection and be application independent.

One way to implement fault tolerant sockets is to use an existing intra-cluster mechanism and make it an intercluster mechanism. This involves relaying network packets between clusters for every network packet received from a client. This transaction must complete before responding to the client. This exasperates the scalability problem. With this type of solution, you minimally double the amount of traffic seen in individual clusters and increase the server response time by the roundtrip latency between clusters. We do not explore this method.

In this paper, we present our implementation of fault tolerant sockets. We propose a method that relies on clientside support. Client-side support consists of a redirection mechanism (client-side redirection), a method to detect communication channel errors to trigger redirection (implicit redirection triggers), and data synchronization (clientside tunnelling). We first introduced the idea of client-side support in [7] where we applied it to connection redirec- tion. We developed RedSocks which uses our session-layer protocol, the Endpoint Operation Protocol (EOP), that allows a server to redirect connections in a flexible, scalable, and application independent way. In this paper, we augment EOP to allow RedSocks to provide communication fault tolerance. Our solution is client transparent, compatible with NAT's and Firewalls, backwards compatible with normal sockets, application independent, handles catastrophic failures, and works with high-performance Internet applications.

In Section 2, we present our fault tolerant socket solution. We follow with a discussion on the details of our implementation. We present experiments, in Section 4, that demonstrate the efficacy of fault tolerant RedSocks. We conclude with a presentation of related work and some parting thoughts.

\section{RedSocks Fault Tolerance}

We have extended BSD socket s with our session layer protocol, EOP, that generates redirection events and enables endpoint redefinition. We call such sockets Redirectable sockets or RedSocks. We looked at explicit redirection events in [6] to increase the scalability of internet applications. We now look at RedSocks and implicit redirection events to construct fault tolerant sockets.

\subsection{Failover Semantics}

We describe the semantics of redirection in RedSocks through a simple example. As shown in Figure 2(a), a communication channel exists between two nodes, $A$ and $B$. An error at $B$ generates an implicit redirection event that changes the "B" endpoint of the channel to $C$ (see Figure 2(b)). $C$ represents a node that may equal $A, B$ or a completely different node. EOP responds to the implicit redirection event by creating a new channel between $A$ and $C$, synchronizing application and communication state, and removing the channel between $A$ and $B$ as seen in Figure 2(c). In Figure 2, a lower-case letter in a box represents

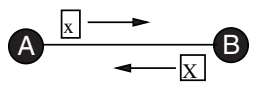

(a) Communication channel

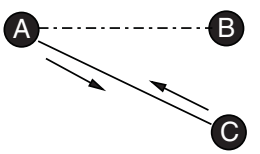

(c) New connection creation

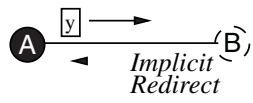

(b) Error Triggers
Redirection Event

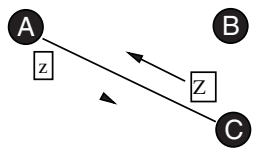

(d) Communication on new channel
Figure 2. Failover from endpoint $B$ to $C$ 


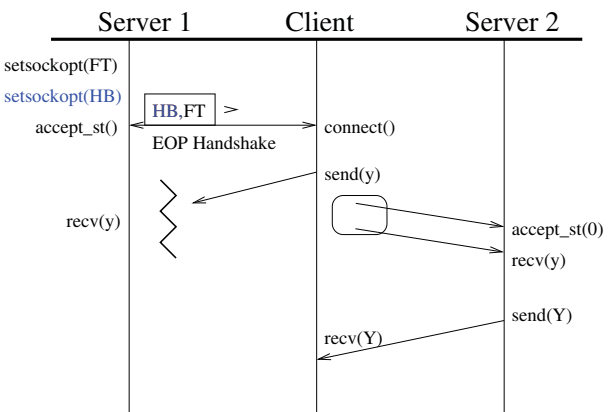

(a) Client Send error without Server Checkpointing

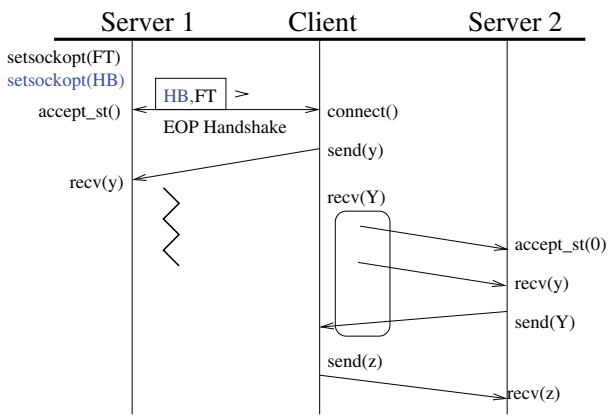

(c) Client Recv error without Server Checkpointing

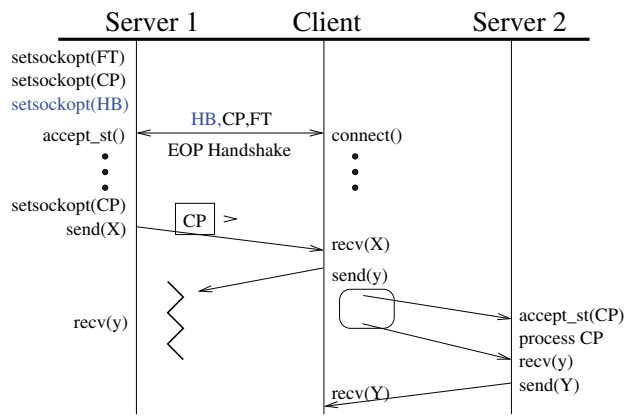

(b) Client Send error with Server Checkpointing

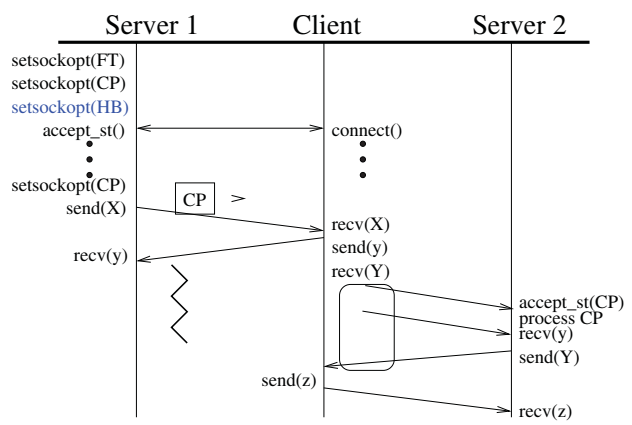

(d) Client Recv error with Server Checkpointing

Figure 3. Implicit Redirection Event Timeline

a request and the corresponding upper-case letter represents the response to that request.

\subsection{Implicit Redirection Events}

Implicit redirection events are generated by errors on the communication channel and serve as triggers for failover. Detecting these errors is not trivial and is explained in Section 3. Implicit redirection is enabled and conditioned via the set sockopt system call. We define three new EOP socket options - heartbeat, checkpoint, and failover list. The EOP heartbeat option starts a heartbeat mechanism, at a rate determined by the server, on the client side of the communication channel. The heartbeat mechanism is necessary for detecting host failure or network partitioning, because normal TCP detection of these errors typically takes well over twenty minutes. The EOP checkpoint option buffers server application state at the client and is used to update the alternate server to which the client is directed. Alternate servers are provided to the client-side EOP by the EOP failover list option. No failover can occur until this option is set by the server. The last component that enables implicit redirection is that the client side EOP layer buffers the last data sent and can resend this data, if necessary, to handle in-flight data.

Our form of failover has several appealing properties. First, our mechanism depends only on communication channel feedback, i.e. TCP errors, and eliminates the need for 3rd party health monitors prevalent in other solutions. Second, the mechanism directly handles state transfer and synchronization. Other solutions, see Section 5, often involve dedicated backup servers or complex communication channel taps that constantly record packet traffic. Finally, by buffering data at the client and being able to redirect across a WAN, ours is the only solution that handles catastrophic failures.

Figure 3 provides a system call level view of host interactions for the different implicit redirection cases. In Figure 3, a lowercase letter denotes a request, an uppercase letter denotes a reply, a rounded box represents EOP processing that is transparent to the client, and 0 indicates a null parameter. In addition, we use $F T$ to denote the fault tolerant list option, $H B$ to denote the heartbeat option, and $C P$ to denote the checkpointing option. The $H B$ option is shaded in the figure. If used, then the jagged line in the figure represents both process failures and network partitioning. Otherwise, it only represents process failures.

Figure 3(a) gives the case where the server is not using the checkpoint option and the error is detected during a send system call at the client. Server 1 must set the $F T$ socket option before accepting the connection. If the server does not, the connection will be vulnerable to errors until the option is set. The server can also set the FT socket 
option at any time in order to update the list of alternate servers. This list, along with all other option settings, is passed to the client during the EOP handshake (see Section 3). The client-side EOP is notified of a communication channel error via TCP error codes or the heartbeat option. It then picks an alternate server from the failover list, creates a new connection to this server, forwards the previous request $y$ to Server 2, and then returns control to the client. Normal communication ensues.

Figure 3(b) only differs from Figure 3(a) in that checkpointing is used. Server 1 updates the checkpointed state as needed. This state usually reflects application layer state associated with serving requests on the communication channel, such as a file name and offset. The checkpointed state is buffered at the client as part of the session layer communication state and overwritten on each update. The error detection and redirection at the client occur as before, except that the checkpointed data is forwarded to Server 2 arriving in an accept_st parameter. The client again forwards the last request. Server 2 sends a reply to this request and continues to serve any additional requests.

Figures 3(c) and 3(d) are the recv error counterparts of Figures 3(a) and 3(b), respectively. While the client is blocked in the recv system call, EOP detects the error, creates a communication channel to an alternate server, forwards the previous request, forwards any checkpointed data, and then continues to wait for a reply. The client has the capability to set an EOP socket option that will return an EREDO error instead of automatically buffering and forwarding the previous request at failover. This allows for more control when the client is EOP-aware.

The servers need to carefully handle data synchronization in persistent storage with implicit redirection. For example, in Figure 3(d) Server 1 could have accomplished various levels of processing on the client's request before failing. If this processing entails updating a persistent store, Server 2 might repeat updates to this store causing data corruption. If such updates are possible, then the servers could commit updates after the request/response transactions completes, have a rollback mechanism invoked when servers fail, or have a 3rd party mechanism to record the partial processing done that Server 2 can query.

\section{Implementation}

There are several different, complimentary strategies for implementing RedSocks. You can use a library, proxy, session layer solution, or RedSocks can be directly incorporated into the application. In our implementation, we chose a session layer solution and include a TCP option to discover the protocol, so that it can be deployed incrementally. Thus, machines can begin aggressively incorporating RedSocks and its functionality can be incrementally used as

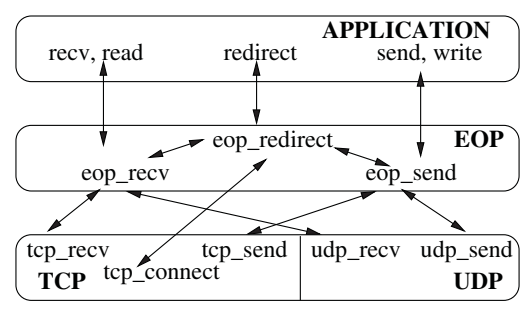

\section{Figure 4. The architecture for EOP and Red- Socks}

peers follow suit.

\subsection{Environment}

We built our fault tolerant sockets in the Linux 2.4.16 kernel, which implements BSD sockets that conform to version 4.4BSD. All code is written in $\mathrm{C}$ and the kernel was compiled with egcs-2.91.66.

\subsection{Architecture}

The fault tolerant socket architecture, shown in Figure 4, is an extension of the architecture we used for RedSocks [7]. We discuss the EOP and transport layer changes necessary to implement our fault tolerant sockets.

\begin{tabular}{|c|c|c|c|c|}
\hline 16 & & 64 & & 104 \\
\hline OPCODE & PORT & IP ADDRES\$ & SLEN & HLEN \\
\hline
\end{tabular}

\section{Figure 5. The new EOP Header}

(a) General Format:

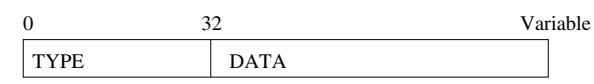

(b) Option format for Backup Server List:

\begin{tabular}{|l|c|c|c|c|c|c|}
\hline 0 & 64 \\
\hline ECP_OPT_FTLIST & Size & IP & Port & IP & Port & ... \\
\hline
\end{tabular}

Figure 6. EOP option formats

\subsubsection{Endpoint Operation Protocol}

We modified the EOP header to allow options. We use the header options to send the backup server list, heartbeat rate, and checkpointed application data necessary for our fault tolerant socket implementation. The new EOP header is given in Figure 5. The additional field hlen is a 8-bit field that provides the length of the EOP header plus the options. Figure 6 illustrates the general EOP option format and the specific format for sending a backup server list. 


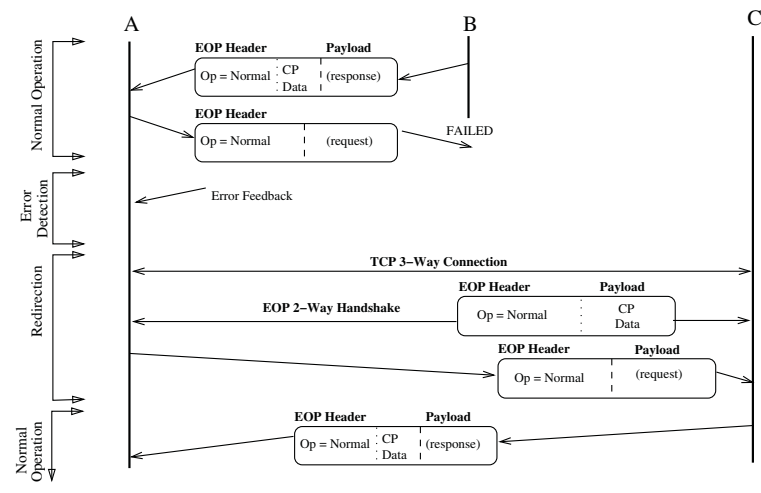

Figure 7. Timeline for EOP packet flow for implicit redirection with a response and application state

Implicit Redirection Time Flow Diagram Figure 7 gives the time flow diagram for an implicit redirection event that includes checkpointed state. $B$ periodically checkpoints its application data during normal communication with $A$. When $B$ fails, EOP receives communication channel feedback indicating an error which triggers redirection. First, EOP chooses an alternate server from the list provided at connection time ${ }^{1}$, connects to it, provides the checkpointed data during the 2-way EOP handshake, and forwards the previous request. Communication between $A$ and $C$ proceeds normally.

Endpoint Failure Detection On the client, the failure of the server-side endpoint is only detectable through errors reported by the transport layer. The EOP layer must capture all relevant error codes to know when to invoke the failover mechanism. The failure mechanism is essentially the RedSocks redirect function with two differences: The redirect function is invoked by error handling code on the client, not by a "redirect" EOP header sent by the server, and its target is selected from the client's backup server list, rather than being pulled from an EOP header.

Table 1 depicts five different failure scenarios. It provides the type of error that is reported to the application, TCP's response, and the fault tolerant action that should be taken for both process and machine failure for each scenario. For network partition failures that separate intermediate routers, we rely on feedback from ICMP messages.

Scenario I describes the errors that can occur during the first connection attempt by the client. If an error occurs at this time, there is no failover action to be taken by the client. In other words, fault tolerance does not apply until a connection is established.

Scenario II, which represents failures that occur during a client send, has two subclasses defined for process fail-

\footnotetext{
${ }^{1}$ The use of the option that provides a alternate server list is not shown in the diagram
}

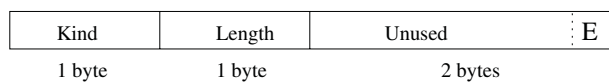

Figure 8. A new TCP option format for socket functionality discovery.

ure. When the server process fails, the server-side kernel responds with a partial close of its end of the socket. The client still sends its message and the receipt of that message generates a TCP reset, denoted RST in the table, from the server-side TCP. If the RST arrives before the client calls recv, then we get case (a) in the table: the error is detected and the failover operation should be invoked. If the RST arrives after the client calls recv, then the client can only detect the sending of FIN which causes the recv call to return with zero bytes read. From the point-of-view of the client, this seems like a normal EOF occurring in the socket stream. To distinguish between these cases, we added an ENDTOKEN that the server sends, in the EOP header, on a normal close. If client receives a FIN but no ENDTOKEN, then the client knows it encountered a failure and should fail over. When the server-side machine fails during scenario II, the client encounters an extremely long ACK timeout ${ }^{2}$. This delay is not transparent to the client and another solution is required to detect this condition. Our solution is to have EOP periodically send heartbeat messages.

In scenario III, process failures are immediately detected and the client fails over. With machine failure, scenario III poses a more serious problem. No error conditions are generated and the client will block on the recv system call indefinitely. Again, we require a heartbeat mechanism to handle this situation.

Last, scenario IV is the connection that occurs during the failover operation. When errors are detected the client tries the next server in the backup server list. If every server on the list is down, then the socket terminates and reports the error to the client.

\subsection{Deployability}

We enhanced our fault tolerant socket implementation by adding a negotiation mechanism that allows both endpoints to discover socket functionality. At connection-time, each end-point checks its peer and discovers whether EOP is supported and adjusts accordingly. We accomplished this by adding a new TCP option that sends the "conditioning" of the socket during the TCP 3-way handshake needed to form a connection. If no option arrives during the handshake, then the remote end is not enabled with our enhanced socket features. Since TCP ignores all unknown options, the impact of sending this option to systems that do not support it is negligible.

\footnotetext{
${ }^{2}$ Twenty three minutes for our Linux 2.4 .16 test
} 


\begin{tabular}{|c|c|c|c|c|c|c|}
\hline \multirow{3}{*}{$\begin{array}{l}\text { FAILURE } \\
\text { SCENARIO }\end{array}$} & \multicolumn{3}{|c|}{ PROCESS FAILURE } & \multicolumn{3}{|c|}{ MACHINE FAILURE } \\
\hline & \multicolumn{2}{|c|}{ FEEDBACK } & \multirow[b]{2}{*}{ ACTION } & \multicolumn{2}{|c|}{ FEEDBACK } & \multirow[b]{2}{*}{ ACTION } \\
\hline & ERROR TYPE & TCP RESPONSE & & ERROR TYPE & TCP RESPONSE & \\
\hline $\begin{array}{l}\text { I. Initial } \\
\text { Connection }\end{array}$ & $\begin{array}{l}\text { connect: refused } \\
\text { connection }\end{array}$ & $\begin{array}{c}\text { server: } \\
\text { RST }\end{array}$ & none & $\begin{array}{l}\text { connect: no } \\
\text { route to host }\end{array}$ & $\begin{array}{l}\text { client: SYN } \\
3 \text { times }\end{array}$ & none \\
\hline IIa. Send & $\begin{array}{c}\text { recv: } \\
\text { broken pipe }\end{array}$ & $\begin{array}{l}\text { server: FIN and } \\
\text { RST before recv }\end{array}$ & failover & \multirow{2}{*}{$\begin{array}{l}\text { recv: no } \\
\text { route to host }\end{array}$} & \multirow{2}{*}{$\begin{array}{l}\text { ACK Timeout } \\
=23 \text { minutes }\end{array}$} & \multirow{2}{*}{$\begin{array}{c}\text { (heartbeats) } \\
\text { failover }\end{array}$} \\
\hline IIb. Send & $\begin{array}{l}\text { recv: returns } 0 \\
\text { no error }\end{array}$ & $\begin{array}{l}\text { server: FIN and } \\
\text { RST after recv }\end{array}$ & $\begin{array}{l}\text { no end token?, } \\
\text { failover }\end{array}$ & & & \\
\hline $\begin{array}{l}\text { III. Recv } \\
\text { (partial data) }\end{array}$ & $\begin{array}{l}\text { recv: connection } \\
\text { reset by peer }\end{array}$ & $\begin{array}{c}\text { both } \\
\text { sent RST }\end{array}$ & failover & none & none & $\begin{array}{c}\text { (heartbeats) } \\
\text { failover }\end{array}$ \\
\hline $\begin{array}{l}\text { IV. Failover } \\
\text { Connection }\end{array}$ & same as I & same as I & failover & same as I & same as I & failover \\
\hline
\end{tabular}

Table 1. TCP error messages and responses for given server failure scenarios with desired fault tolerant socket behavior.

Figure 8 shows the format for this option. The kind field identifies the option. The length field equals the total length of the option, which is four bytes in this case. The last two bytes comprise a bitmask indicating what features are supported. At this time, we only have one feature - EOP enabled (E).

\section{Experiments}

We first measured the overhead of using our fault tolerant sockets when no failure occurs using two 400Mhz dualprocessor Pentium III machines. We measured the normal communication exchange between our custom client/server applications with our heartbeat mechanism enabled for different sizes of data to checkpoint. The checkpointing scheme we employ is to checkpoint application state before every send system call. This simulates the situation where every request is for a file to download which typically occurs in ftp and web servers. We show the client latency for reply/request exhanges, varying in size from 100 bytes to 32K, in Figure 9(a). To serve as a basis for comparison, we include the results for communication with heartbeats and checkpointing turned off; the line labeled "Normal" represents these results. As Figure 9(a) clearly shows, heartbeats and checkpointing add minimal overhead.

For our failover overhead measurements, we used three 400Mhz dual-processor Pentium III machines. The server provides the client with a list of alternate servers to failover to when the client connects via the EOP handshake. At some random point in the communication with the client, the server aborts the connection. When the client-side EOP detects the failure, it selects an alternate server from the failover list provided by the original server and opens a connection to it. During the EOP handshake, the client-side EOP provides the alternate server-side EOP with the checkpointed data, the number of bytes read since the check- pointed data arrived, and any data leftover in the client's send buffer. This information is passed up to the alternate server via the accept_st system call. The alternate server parses this data and continues to serve the request. The entire transaction is transparent to the client.

Figure 9(b) shows our results for this scenario for different simulated file sizes and checkpoint data size. Again, we include the results of communication without failure, heartbeats or checkpointing for comparison; these results are represented by the line labeled "Normal" in the graph. We wanted to study the impact of failure on both large and small files. To simulate a large file transfer, we performed 100 hundred send/recv exhanges of the sizes indicated by the x-axis of Figure 9(b). Simarly, we simulated a small file with 5 such exchanges. For a large files, the time associated with failover is amortized across $100 \mathrm{send} / \mathrm{recv}$ exhanges and thus exhibits less overhead than that experienced by small files.

\section{Related Work}

Fault Tolerant RedSocks is the only applicationindependent solution that handles catastrophic failures. We briefly describe application-independent approaches that handle other server-side failures and place our work within the context of these approaches.

The Stream Control Transport Protocol [5] proposes the notion of multi-homing, in which an end-point can be associated with multiple IP-addresses. Upon a network failure, the protocol arranges for data to be sent over to an alternate network path to the same server endpoint. Our mechanism also handles network failure by allowing failover to an alternate network path between the same server endpoint or a different one.

HydraNet-FT [11] uses a redirector for detecting failures and re-mapping an existing connection to a secondary 


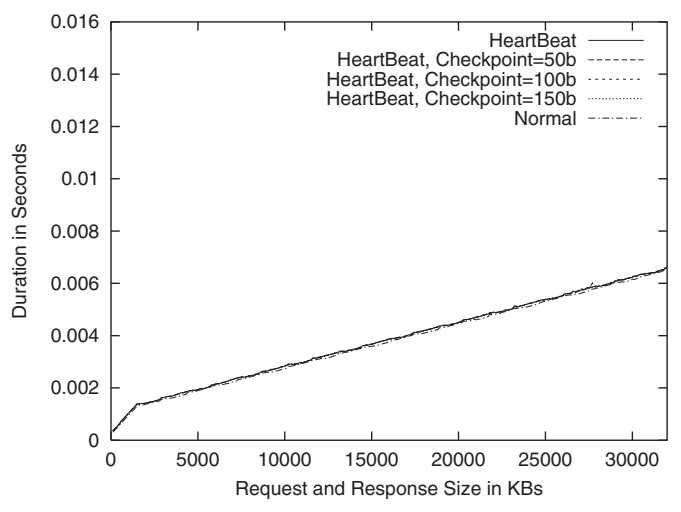

(a) Communication Overhead

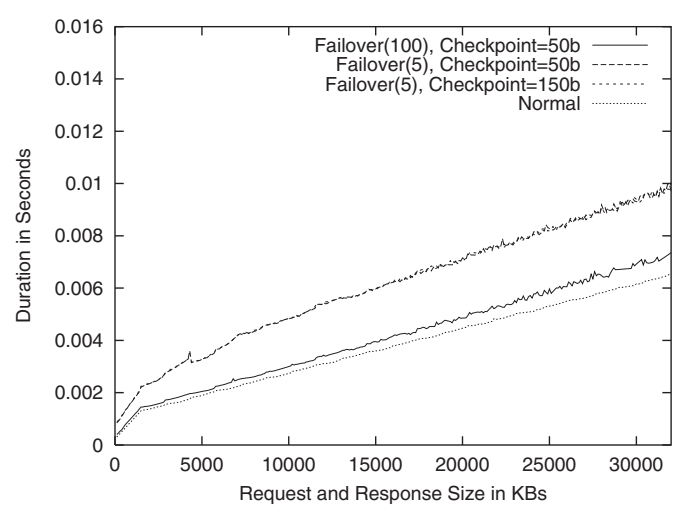

(b) Failover

\section{Figure 9. Failover measurements}

server. The redirector is aware of all replicas, and redirects each client request to a primary and all its replicas. The system, thus, uses a primary-backup scheme. Our approach does not require dedicated replicas.

Alvisi et. al [1] describe a fault tolerant TCP (FT-TCP) in which a failed TCP connection can be restored to a specific communication state after a server recovers from failure. FT-TCP is implemented by a wrapper that checkpoints connection state and data at a separate logger. During the recovery process, the wrapper and the loggers interact to bring the application to a specific state. Our recovery process does not require servers to replay all previous communication events to rebuild communication and application state.

Snoeren et. al [12] propose a connection failover mechanism to provide fault tolerance for a collection of Internet servers. In this approach, each connection is associated with a set of LAN-connected servers. The servers periodically synchronize their connection states. Upon failure, the servers in the group contact the client, which then selects one of the servers to resume connection. Because the server resumes communication, the solution is not compatible with NATs or Firewalls. Our approach differs from [12] in that our notion of connection failover is client-centric. Once a connection fails, a client endpoint determines the server to which it should re-connect. This makes our approach compatible with both NATs and firewalls.

\section{Conclusion}

Fault tolerant RedSocks bring reliability to Internet Applications. Ours is the first, general-purpose solution that handles all server-side failures, including catastrophic failures, in a scalable manner. In future work, we plan to incorporate connection redirection, instream redirection, and fault tolerance to provide a single solution to Internet application scalability and reliability requirements.

\section{References}

[1] L. Alvisi, T. Bressoud, A. El-Khashab, K. Marzullo, and D. Zagorodnov. Wrapping server-side TCP to mask connection failures. In INFOCOM 2001, pages 329-338, Anchorage, AK, April 2001.

[2] N. Bhatti, A. Bouch, and A. Kuchinsky. Integrating userperceived quality into web server design. In WWW Conference, Amsterdam, The Netherlands, 2000.

[3] T. Brisco. DNS support for load balancing. RFC 1794, Rutgers University, April 1995.

[4] Scaling the Internet web servers. www.cisco.com/warp/public/751/lodir/scale.wp.htm, 1997.

[5] R. S. et al. Stream Control Transmission Protoco. IETF, rfc 2960 edition, Oct 2001. http: //www.ietf.org/rfc/rfc2960.txt.

[6] M. Haungs. Providing network programming primitives for internet application construction. Technical report, UC Davis, September 2002.

[7] M. Haungs, R. Pandey, E. Barr, and J. F. Barnes. A fast connection-time redirection mechanism for internet application scalability. In HIPC 2002, Bangalore, India, Dec 2002.

[8] K. Li and B. Moon. Distributed cooperative apache web server. In WWW Conference, pages 555-564, Hong Kong, May 2001.

[9] M. Luo and C. Yang. Constructing zero-loss web services. In Infocom 2001, Anchorage, Alaska, April 2001.

[10] M. Reene. The customer expectation gap. 2002 Enterpulse Survey, www.enterpulse.com.

[11] G. Shenoy, S. Satapati, and R. Bettati. Hydranet-ft: Network support for dependable services. In ICDCS 2000, pages 699706, Taipai, April 2000. IEEE.

[12] A. Snoeren, D. Andersen, and H. Balakrishnan. Fine-grained failover using connection migration. In USITS '01, pages 221-232, San Francisco, CA, March 2001.

[13] V. Zandy and B. Miller. Reliable sockets. http://citeseer.nj.nec.com/zandy01reliable.html. 